Monatsschr Kinderheilkd 2018 · 166:767-773 https://doi.org/10.1007/s00112-018-0531-1

Online publiziert: 10. Juli 2018

(c) Der/die Autor(en) 2018

\section{Redaktion}

R. Kerbl, Leoben

K. Schmitt, Linz

CrossMark

\title{
C. Prandstetter
}

Kepler Universitätsklinikum, Linz, Österreich

\section{Interventionelle Kinder- kardiologie - Entwicklungen, Trends und Grenzen}

und wesentlichsten Trends in der interventionellen Kinderkardiologie. ren zu den häufigsten angeborenen Fehlbildungen. Circa jedes 100. Baby kommt mit einem AHF auf die Welt. Abgesehen von den sehr komplexen AHF-Formen haben die betroffenen Neugeborene heutzutage i. Allg. eine sehr hohe Chance auf ein Leben mit guter Lebensqualität.

\section{Hintergrund}

In den letzten 20 Jahren hat es in der interventionellen Kinderkardiologie wesentliche Fortschritte gegeben: Fetale Interventionen ermöglichen, das Outcome der $\mathrm{Pa}$ tienten bei vorliegenden AHF bereits vor der Geburt zum Positiven zu beeinflussen. Die 3D-Rotationsangiographie vereinfacht komplexe interventionelle Eingriffe, und die Einführung der interventionellen Klappenimplantation im letzten Jahrzehnt war ein weiterer Meilenstein in der Entwicklung der interventionellen Kinderkardiologie. Hybrideingriffe, bei denen Kinderherzchirurg und Kinderkardiologe die Patienten gemeinsam im Hybrid-OP versorgen, ermöglichen neue, individuelle, maßgeschneiderte Problemlösungen.

Die häufigsten Maßnahmen im Herzkatheterlabor betreffen Verschlüsse von Atriumseptumdefekten (ASD), Eingriffe am persistierenden Ductus arteriosus (PDA), im Aortenisthmus, an den Pulmonalarterien (PA) sowie an der Aorten- und Pulmonalklappe [21]. Der vorliegende Beitrag bietet hinsichtlich der häufigsten Eingriffe eine Übersicht über die aktuelle Situation, den State of the Art, die Grenzen sowie die Entwicklung

\section{Verschlüsse}

\section{Atriumseptumdefekt}

Seit der ersten interventionellen Versorgung eines ASD (• Abb. 1) vor mehr als 40 Jahren werden heutzutage ca. drei Viertel aller im Bereich der Fossa ovalis liegender Vorhofseptumdefekte (ASDII) im Herzkatheterlabor verschlossen. Komplikationen und Mortalität sind im Vergleich mit chirurgischen Interventionen geringer [7]. Seit 2011 existieren klare Richtlinien [12] über die Durchführung eines interventionellen ASDVerschlusses. Dennoch gibt es hinsichtlich Indikation, Patientenalter und dem Vorgehen bei derartigen Eingriffen relevante Unterschiede zwischen den einzelnen Institutionen [23].

\section{》) Retroaortaler Rand $<5 \mathrm{~mm}$ stellt relative Kontraindikation für interventionellen ASD-Verschluss dar}

Der mediane Altersdurchschnitt der Patienten beträgt meist etwa 5 bis 7 Jahre. Das größte Komplikationsrisiko hinsichtlich eines interventionellen ASD-Verschlusses besteht in der Erosion durch das ,device“, die auch noch Jahre nach Durchführung des ASD-Verschlusses auftreten kann und der Häufigkeit mit ca. 0,3\% zu beziffern ist [32]. Auch wenn generell ein geringer aortaler Rand und „oversizing“ des Device als Risikofaktoren an- geführt werden, ist es schwierig, Risikopatienten klar zu definieren, da der Mechanismus der Entstehung weiterhin unklar bleibt. Die Erosionsproblematik führte zur Änderung der Empfehlungen zum interventionellen ASD-Verschluss: Ein retroaortaler Rand von weniger als $5 \mathrm{~mm}$ wird nun als relative Kontraindikation für einen ASD-Verschluss angeführt. Ein solcher grenzwertiger Rand liegt jedoch mit einer Prävalenz von ca. 50\% aller Kinder, die zum ASD-Verschluss zugewiesen werden, vor. Die Änderung der Empfehlung bewirkte in den USA bereits, dass wieder vermehrt Kinder zum operativen ASD-Verschluss eingeteilt werden [22]. Ob neuere am Markt verwendete Verschlussmechanismen in Zukunft ein geringeres Erosionsrisiko mit sich bringen werden, kann anhand der mangelnden Datenlage noch nicht sicher beurteilt werden.

\section{Ventrikelseptumdefekt}

\section{Perimembranöse Variante}

Der Großteil der Ventrikelseptumdefekt (VSD), die verschlossen werden müssen, liegt perimembranös (pmVSD). Der erste interventionelle VSD-Verschluss wurde 1988 von Lock et al. beschrieben [19]. Mittlerweise erzielt das interventionelle Vorgehen den chirurgischen Ergebnissen vergleichbare Resultate [26]. Im Jahr 2007 ermittelte allerdings eine retrospektive Multizenterstudie aus Europa eine im Vergleich zu den chirurgischen Ergebnissen insgesamt zu hohe, inakzeptable Atrioventrikular(AV)-BlockRate von 4,8\% [8]. Ebenfalls zu beachtende Probleme stellen eine mögliche Beeinträchtigung der Aorten- und der Tri- 


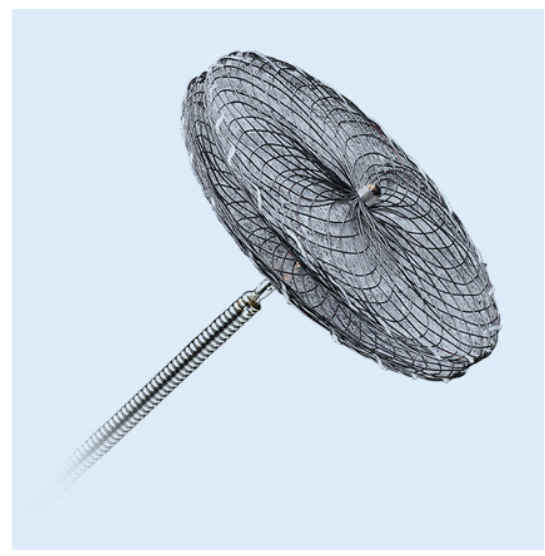

Abb. 1 a Beispiel für den Verschlussmechanismus eines Atriumseptumdefekts: AMPLATZER ${ }^{\text {TM }}$ Septal Occluder. (Mit freundl. Genehmigung, $\odot$ Abbott, Chicago, IL, USA, alle Rechte vorbehalten)

kuspidalklappe (ca. 2,6\% der Fälle) sowie eine nicht zu vernachlässigende RestShunt-Rate (ca. 3,1 \% der Fälle; [33]) dar. Eine Hämolyse durch das Device kann in seltenen Fällen dessen operative Entfernung notwendig machen [24]. Somit ist das chirurgische Vorgehen an vielen Zentren weiterhin bei die bevorzugte Methode des Verschlusses perimembranöser VSD. Die extrem geringe Mortalität und das sehr niedrige AV-Block-Risiko unter $1 \%$ [30] werden auch zukünftig Maßstäbe für den interventionellen VSD-Verschluss bleiben. Die Weiterentwicklung von neuen Verschlussmechanismen (Nit-Occlud ${ }^{\circledR}$ Lê VSD, mVSDOccluder ...) wird weitere Verbesserungen für den interventionellen Verschluss mit sich bringen.

\section{Muskuläre Varianten}

Auch wenn die meisten muskulären Ventrikelseptumdefekte (mVSD, - Abb. 2) klein sind oder sich spontan verschließen, gibt es immer wieder solche, die verschlossen werden müssen. Im Vergleich zu den pmVSD ist der chirurgische Verschluss meist ungleich schwieriger, da der VSD für den Chirurgen über den üblichen transtrikuspidalen Zugangsweg im Trabekelwerk des rechten Ventrikels oft nur schwer zu finden ist. Daraus resultieren eine deutlich höhere RestShunt-, Komplikations- und auch Mortalitätsrate (einstelliger Prozentbereich)

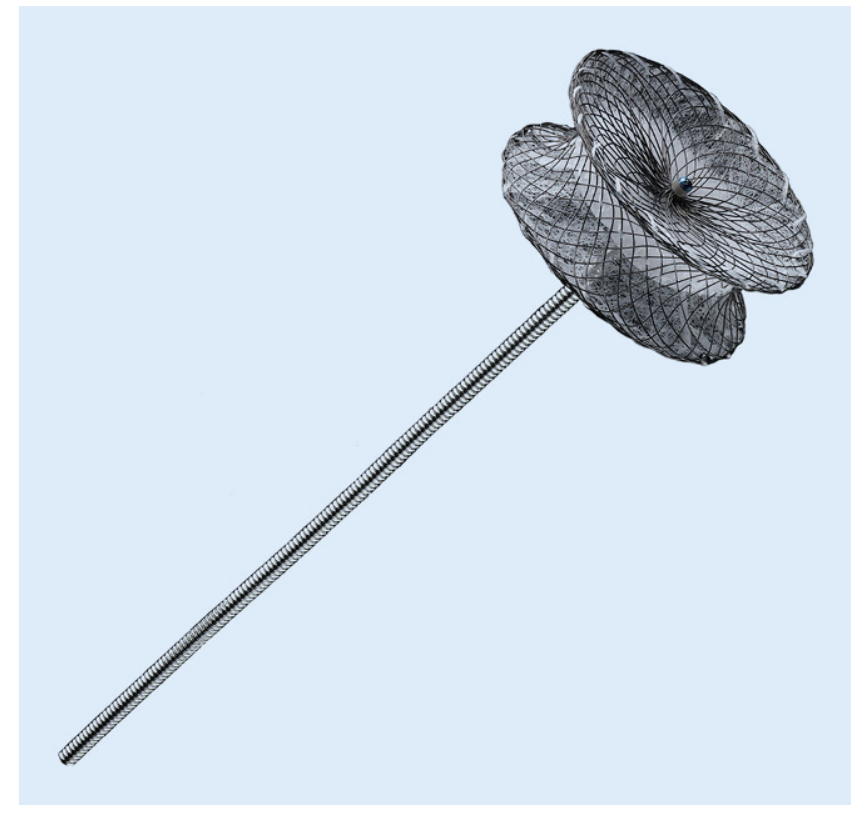

Abb. $2 \triangleleft$ Beispiel für den Verschlussmechanismus eines muskulären Ventrikelseptumdefekts: AMPLATZER ${ }^{\text {TM }}$ Muscular VSD Occluder. (Mit freundl.Genehmigung, $\odot \mathrm{Fa}$. Abbott, Chicago, IL, USA, alle Rechte vorbehalten)

als beim pmVSD. Trotz der derzeitigen allgemeinen Tendenz zum frühzeitigen operativen VSD-Verschluss kann insbesondere bei kritisch kranken und sehr kleinen Neugeborenen das PA-Banding eine sinnvolle Alternative sein. Eine attraktive Entwicklung in diesem Zusammenhang stellt das selbstabsorbierbare PA-Banding dar [10].

Für größere, ältere Säuglingen und Kleinkinder stellt der interventionelle Verschluss eine mittlerweile sichere und gute Alternative zum chirurgischen Vorgehen dar. Bei sehr kleinen Patienten $(<5 \mathrm{kgKG})$ bietet aufgrund vaskulärer Limitationen und technischer Schwierigkeiten der periventrikuläre Hybrideingriff ein gutes Lösungsmodell [34]. Hierbei kann der VSD nach Sternotomie mithilfe transösophagealer oder perikardialer Echokardiographie am schlagenden Herzen dargestellt und anschließend über eine direkte transkardiale Schleuse verschlossen werden.

\section{Persistierender Ductus arteriosus}

Mit 5-10\% ist der PDA einer der häufigsten angeborenen Herzfehler. Heute besteht ein allgemeiner Konsens, dass große, hämodynamisch relevante PDA verschlossen sowie kleine unbedeutende PDA offen gelassen werden können („silent duct“; [2]). Chirurgische Verschlüsse haben selbst bei Frühgeborenen mit $<1000$ gKG und $<28$ Gestationswochen $(\mathrm{GW})$ eine hohe Erfolgsquote (nahezu $100 \%)$ mit geringer Mortalitätsrate $(<2 \%,[1])$.

》) Katheterintervention ist
primäre PDA-Verschlussmethode
bei Säuglingen und Kindern
$>3-5$ kgKG

Im Jahr 1967 gelang es Portsman et al. erstmals, einen PDA interventionell $\mathrm{zu}$ verschließen [28]. Während anfänglich noch sehr große Schleusen (beachte: $13-28 \mathrm{~F}$ ) benötigt wurden, hat sich mittlerweile die Schleusengröße auf 3-4F reduziert, sodass die Katheterintervention die primär bevorzugte Verschlussmethode bei Säuglingen und Kindern $>3-5 \mathrm{kgKG}$ geworden ist. Die am häufigsten eingesetzten Verschlussmechanismen sind Coils (• Abb. 3) und Occluder (• Abb. 4).

Weitere Entwicklungen, wie z.B. der erst kürzlich auf den Markt gekommene AMPLATZER ${ }^{\mathrm{Tm}}$ Duct Occluder II Additional Sizes (ADOIIAS), ermöglichen es zunehmend, PDA auch bei Neu- und Frühgeborenen $<3 \mathrm{~kg}$ mit sehr guten Ergebnissen mithilfe der interventionellen Methode zu verschließen. Teilweise werden die Eingriffe direkt auf der Neugeborenenintensivstation ohne Durchleuch- 
Hier steht eine Anzeige.

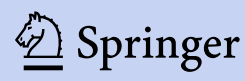


tung durchgeführt. Es gibt Studien, die eine geringere Morbidität und raschere Erholung als nach einem chirurgischen Eingriff belegen [25]. Dem steht eine jedoch nicht zu negierende Rate an Embolisationen und vaskulären Komplikationen gegenüber $[3,16]$.

\section{Stents und Dilatationen}

Die häufigsten Lokalisationen für eine Stent-Implantation sind der Aortenisthmus sowie die PA bzw. der PA-Hauptstamm (meist bei Patienten mit M. Fallot und Varianten). Als eine weitere relevante Lokalisation für Stents in der Kinderkardiologie ist der Ductus arteriosus (ca. 14\% der Fälle) zu nennen. In der französischen Multizenterstudie von Hascoët et al. [14] verliefen diese Eingriffe in fast $97 \%$ der Fälle erfolgreich, aber die periprozedurale Mortalitätsrate betrug ca. 1,3\%, und in 14,3\% Fälle traten Komplikationen, hiervon ein Viertel vaskulärer Art, auf. Die Komplikationsrate war insbesondere bei PDA-Stenting und „prestenting“ vor Pulmonalklappenimplantation am höchsten.

\section{Pulmonalarterien-Stenting}

Ein Eingriff an den PA findet zu 20\% bei Kindern im Alter unter einem Jahr statt, zu $67 \%$ bei Kindern zwischen dem ersten und dem 18. Lebensjahr sowie bei nur ca. $13 \%$ der Kinder im Alter darüber (Lewis et al. [17]). In ca. zwei Drittel der Fälle ist die linke PA betroffen. In ihrer Multizenterstudie ermittelten Lewis et al. eine Erfolgsrate von ca. $76 \%$. Die Komplikationsrate betrug ca. $14 \%$ und die nicht ganz zu negierende Mortalitätsrate $1,7 \%$. Die meisten Komplikationen traten bei Kindern mit einem Körpergewicht unter $4 \mathrm{~kg}$, bei Single-ventricle-Patienten sowie im Rahmen von Notfalleingriffen auf. Auch wenn mithilfe verbesserter Diagnostik (MRT, 3D-Rotationsangiographie) selbst komplexe Bifurkationsstenosen interventionell behandelt werden können, sollte nicht vergessen werden, dass die chirurgische Sanierung manchmal die bessere Alternative sein kann.

Monatsschr Kinderheilkd 2018 $\cdot 166: 767-773$ https://doi.org/10.1007/s00112-018-0531-1

(c) Der/die Autor(en) 2018

\section{Prandstetter}

\section{Interventionelle Kinderkardiologie - Entwicklungen, Trends und Grenzen}

\section{Zusammenfassung}

In den letzten 20 Jahren hat es in der interventionellen Kinderkardiologie wesentliche Fortschritte gegeben. Dieser Beitrag beschreibt die aktuelle Situation bezüglich der am häufigsten durchgeführten Eingriffe im pädiatrischen Herzkatheterlabor. Aufgrund zahlreicher neuer Entwicklungen sind heutzutage bereits sehr viele Probleme von Patienten mit angeborenem Herzfehler (AHF) im Herzkatheterlabor lösbar. Aber nicht immer muss der Herzkathetereingriff die bessere Alternative zur Operation darstellen. Nur durch gute Zusammenarbeit aller beteiligter Disziplinen (Diagnostik, Intervention, Kinderherzchirurgie) kann die für den Patienten beste Lösung gefunden werden.

\section{Schlüsselwörter}

Angeborene Störungen · Herzkatheterisierung · Stents - Dilatation . Interventioneller Verschluss

\section{Pediatric interventional cardiology—Development, trends and limitations}

\section{Abstract}

Significant progress has been made in pediatric interventional cardiology over the past 20 years. This article provides a brief overview of the current situation, state of the art and key trends in pediatric interventional cardiology with respect to the most commonly performed procedures. Nowadays, many problems in congenital heart disease can be managed in the catheter laboratory; however, cardiac catheterization is not always the better alternative to surgery. Good cooperation of all participating disciplines (diagnostics, intervention, pediatric cardiac surgery) is essential to find the best solution for the patient.

\section{Keywords}

Congenital disorders - Heart catheterization . Stents · Dilatation · Interventional closure

\section{Aortenisthmusstenose}

Ungefähr 5-7\% aller AHF betreffen eine Aortenisthmusstenose. Im Jahr 1982 gelang es Lock et al. erstmals, eine Aortenisthmusstenose interventionell zu dilatieren [18]. Seit 1991 werden Stents verwendet und seit 1999 gecoverte Stents. Insbesondere für ältere Kinder und Jugendliche sowie Erwachsenen stellt die Stent-Implantation mittlerweile die Methode der Wahl dar. Fast immer kann ohne nennenswerte Reinterventionsraten [15] eine ausreichende Reduktion des Druckgradienten im Katheterlabor erreicht werden.

Die Ballonangioplastie kommt in erster Linie bei Restenosen nach chirurgischen Eingriffen zu Einsatz. Diese wird derzeit an den meisten Zentren (noch?) bei Neugeborenen und Säuglingen anstelle des Kathetereingriffs durchgeführt.
Vaskuläre Zugangsprobleme sowie fehlende implantierbare Stents, die bis auf Erwachsenengröße dilatiert werden können, stellen nach wie vor das größte Hindernis für den interventionellen Zugang dar. Brechbare [35] oder auch bioadsorbierbare [29] Stents werden evtl. zukünftig auch in diesen Fällen eine Alternative zur Operation werden.

\section{Duktus-Stents}

Erstmals 1992 wurde über ein DuktusStenting als Alternative zum systempulmonalarteriellen Shunt von Gibbs et al. berichtet [13]. Obwohl anfängliche Ergebnisse nicht vielversprechend waren, ist der Duktus-Stent inzwischen durch verbesserte Techniken eine Alternative zum chirurgischen Eingriff bei Singleventricle-Physiologie geworden. Hier gewährleistet er die Aufrechterhaltung 


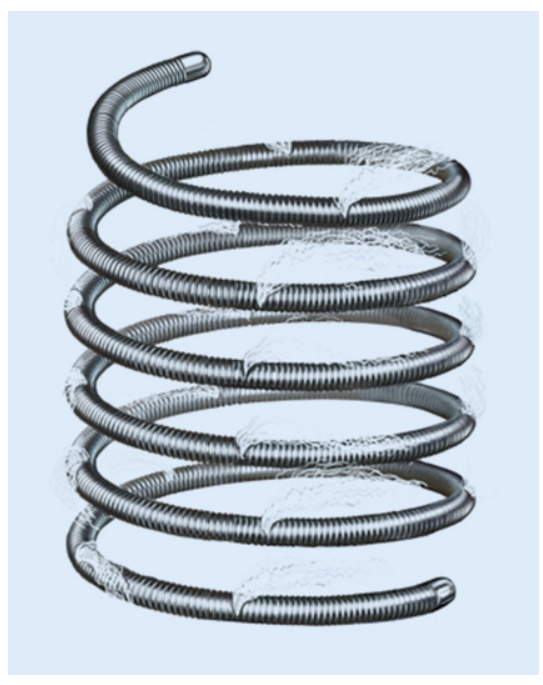

Abb. 3 A Beispiel für einen Coil: Multi Loop Coil (Mit freundl. Genehmigung, ( ) Fa. Boston Scientific, Marlborough, MA, USA, alle Rechte vorbehalten)

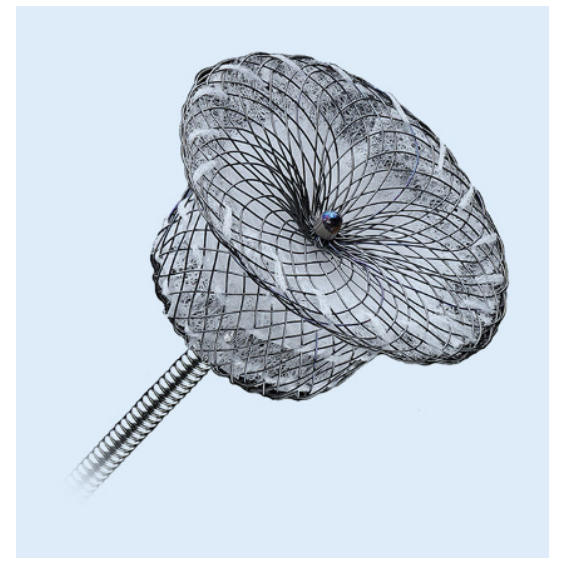

Abb. 4 a Beispiel für den Verschlussmechanismus eines persistierenden Ductus arteriosus: AMPLATZER ${ }^{\text {TM }}$ Duct Occluder. (Mit freundl. Genehmigung, $\odot$ Fa. Abbott, Chicago, IL, USA, alle Rechte vorbehalten)

\section{Klappenimplantationen}

Ungefähr $20 \%$ aller AHF benötigen wiederholte Eingriffe an der Pulmonalklappe. Mit der ersten Implantation einer Pulmonalklappenprothese (Melody ${ }^{\mathrm{mix}}$; - Abb. 5) zur Jahrhundertwende schuf Bonhoeffer die Möglichkeit, diesen Eingriff auch interventionell durchzuführen [6]. Die Melody ${ }^{\mathrm{m}}-$ Prothese besteht aus einer Rinderjugularvenenklappe, die in einen Platinum-Iridium-Stent eingenäht ist. Das größte Risiko der perkutanen Pulmonalklappenprothesenimplantation stellt bei ca. $5 \%$ der Patienten die Kompression der Koronararterien dar. Mithilfe des ,prestenting“(Stent-Implantation direkt vor der Klappenimplantation) konnten Risiken, wie Stent-Fraktur und Ruptur des Conduits, deutlich minimiert werden. Weitere Komplikationen bestehen in einer nicht zu negierenden, etwas höheren Endokarditisrate (2-6\% [20]) und in sehr seltenen Fällen in der Ruptur eines meist kalzifizierten Homografts. Für eine Klappenimplantation zu weit dilatierte rechtsventrikuläre Ausflusstrakte und vaskuläre Zugangsprobleme bei einer derzeit notwendigen Schleusengröße von $22 \mathrm{~F}$ sind ebenfalls Limitationen.

\section{》) „Prestenting“ mindert Risiken wie Stent-Fraktur und Conduit- Ruptur} chen Zentren auch der systemarteriellen Zirkulation (hypoplastisches Linksherzsyndrom: „Giessen procedure“). Insbesondere bei duktusabhänigigem PA-Flow zeigt der Stent Vorteile gegenüber dem chirurgischen Eingriff, bei allerdings etwas erhöhten Reinterventionsraten [5].

\section{Klappeninterventionen}

\section{Klappendilatationen}

Seit den ersten Klappendilatationen ist die interventionelle Aorten- und Pulmonalklappensprengung die $\mathrm{Me}$ thode der Wahl zur Behandlung von stenotischen Semilunarklappenvitien geworden. Während v. a. die Pulmonalklappensprengung bei Patienten nach dem 1. Lebensjahr exzellente Ergebnisse

vorweisen kann [27], erfolgt der Eingriff an Aortenvitien meist palliativ und wird durchgeführt, um ein möglichst langes Intervall zum Aortenklappenersatz zu erzielen. Soulatges et al. [31] bezifferten die Operationsfreiheit 5, 10 und 20 Jahre nach Aortenklappendilatation mit 82, 72 bzw. $66 \%$. Vor allem bei grenzwertig kleinen Ventrikeln mit nur kleinem Aortenklappendurchmesser sollte aber eine neonatale Ross-Operation in Erwägung gezogen werden. Während Studien existieren, die dem chirurgischen und dem interventionellen Verfahren ähnliche Ergebnisse bescheinigen [31], gibt es in den letzten Jahren kritische Stimmen, die meinen, dass verbesserte Techniken der Klappenrekonstruktionschirurgie bessere Ergebnisse ermöglichen könnten [4, $11]$.
Die Melody ${ }^{\text {mm }}$-Prothese wurde aber auch schon in Trikuspidal-, Mitral- und bei Patienten mit Fontanphysiologie sogar in Neoaortenposition entweder in Form von perkutanen Interventionen oder $\mathrm{Hy}$ brideingriffen mit guten Ergebnissen implantiert [9]. Neue Entwicklungen wie z. B. das native Outflow Tract Device (Fa. Medtronic, Dublin, Irland), die Venus P Valve (Fa. Venus Medtech, Hanzhou, China) und SAPIEN XT (Fa. Edwards Lifescience, Irvine, CA, USA) werden hoffentlich weiteren Patienten die Notwendigkeit einer chirurgischen Lösung ersparen. Vermutlich ist es ebenso nur noch eine Frage der Zeit, bis auch eine interventionelle Aortenklappenprothesenimplantation für Kinder verfügbar sein wird. 


\section{Fazit für die Praxis}

\section{- Aufgrund der Entwicklung immer kleinerer Schleusen, diverser neuer Verschlussmechanismen und der Möglichkeit, Klappen interventionell zu implantieren, sind heutzutage sehr viele mit AHF einhergehende Probleme im Herzkatheterlabor lösbar. \\ - Nicht immer ist der Herzkatheter- eingriff die bessere Alternative zur chirurgischen Operation. Unab- hängig von allgemeinen Trends, Richtlinien und Entwicklungen wird stets die beste Lösung für den ein- zelnen Patienten nur durch gute Zusammenarbeit zwischen den ein- zelnen Subdisziplinen in Abwägung der eigenen vorhanden Ressourcen und Expertise gefunden.}

\section{Korrespondenzadresse

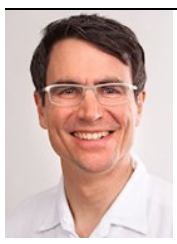 \\ Dr. C. Prandstetter \\ Kepler Universitätsklinikum Krankenhausstr. 26, 4020 Linz, Österreich christoph.prandstetter@ kepleruniklinikum.at}

Funding. Open access funding provided by Johannes Kepler University Linz.

\section{Einhaltung ethischer Richtlinien}

Interessenkonflikt. C. Prandstetter gibt an, dass kein Interessenkonflikt besteht.

Dieser Beitrag beinhaltet keine von den Autoren durchgeführten Studien an Menschen oder Tieren.

Open Access. Dieser Artikel wird unter der Creative Commons Namensnennung 4.0 International Lizenz (http://creativecommons.org/licenses/by/4.0/deed. de) veröffentlicht, welche die Nutzung, Vervielfältigung, Bearbeitung, Verbreitung und Wiedergabe in jeglichem Medium und Format erlaubt, sofern Sie den/die ursprünglichen Autor(en) und die Quelle ordnungsgemäßnennen, einen Linkzur Creative Commons Lizenz beifügen und angeben, ob Änderungen vorgenommen wurden.

\section{Literatur}

1. Avsar MK, Demir T, Celiksular C, Zeybek C (2016) Bedside PDA ligation in premature infants less than 28 weeks and 1000 g. JCardiothorac Surg 11(1):146

2. Baruteau A-E, Hascoët $S$, Baruteau J, Boudjemline Y, Lambert V, Angel C-Y et al (2014) Transcatheter closure of patent ductus arteriosus: past, present and future. Arch Cardiovasc Dis 107(2):122-132

3. Baspinar O, Sahin DA, Sulu A, Irdem A, Gokaslan G, Sivasli E, Kilinic M (2015) Transcatheter closure of patent ductus arteriosus in under $6 \mathrm{~kg}$ and premature infants. J Interv Cardiol 28(2):180-189

4. Benson L (2016) Neonatal aortic stenosis is a surgical disease: an interventional cardiologist view. Semin Thorac Cardiovasc Surg Pediatr Card Surg Annu 19(1):6-9

5. Bentham JR, Zava NK, Harrison WJ, Shauq A, Kalantre A, Derrick G et al (2018) Duct stenting versus modified Blalock-Taussig shunt in neonates with duct-dependent pulmonary blood flow. Circulation 137(6):581-588

6. Bonhoeffer $P$, Boudjemline $Y$, Saliba $Z$ et al (2000) Percutaneous replacement of pulmonary valve in a right-ventricle to pulmonary-artery prosthetic conduit with valve dysfunction. Lancet 356(9239):1403-1405

7. Butera G, Biondi-Zoccai G, Sangiorgi G et al (2011) Percutaneous versus surgical closure of secundumatrial septal defects: a systematic review and metaanalysis of currently available clinical evidence. Eurolntervention 7:377-385

8. Carminati M, Butera G, Chessa M, De Giovanni J, Fisher G, Gewillig M, Peuster M, Piechaud JF, Santoro G, Sievert H, Spadoni I, Walsh K, Investigators of the European VSD Registry (2007) Transcatheter closure of congenital VSDs: results of the European Registry. Eur Heart J 28:2361-2368

9. Cullen MW, Cabalka AK, Alli OO, Pislaru SV, Sorajja P, Nkomo VT, Malouf JF, Cetta F, Hagler DJ, Rihal CS (2013) Transvenous, antegrade melody valvein-valve implantation for bioprosthetic mitral and tricuspid valve dysfunction: a case series in children and adults. JACC Cardiovasc Interv 6(6):598-605

10. Daley M, Brizard CP, Konstantinov IE, BrinkJ, Jones B, d'Udekem Y (2017) Absorbable pulmonary artery banding: a strategy for reducing reoperationst. Eur J Cardiothorac Surg 51(4):735-739

11. Donald JS, Konstantinov IE (2016) Surgical aortic valvuloplasty versus balloon aortic valve dilatation in children. World J Pediatr Congenit Heart Surg 7(5):583-591

12. Feltes TF, Bacha E, Beekman RH et al (2011) Indications for cardiac catheterization and intervention in pediatric cardiac disease: a scientific statement from the American Heart Association. Circulation 123:2607-2652

13. Gibbs JL, Rothman MT, Rees MR, Parsons JM, Blackburn ME, Ruiz CE (1992) Stenting of the arterial duct: A new approach to palliation for pulmonary atresia. BrHeart J 67(3):240-245

14. Hascoët S, Jalal Z, Baruteau A, Mauri L, Chalard A Bouzguenda I et al (2018) Stenting in paediatric and adult congenital heart diseases: a French multicentre study in the current era. Arch Cardiovasc Dis 108(12):650-660

15. Holzer R, Qureshi S, Ghasemi A, Vincent J, Sievert H, Gruenstein D, Weber H, Alday L, Peirone A, Zellers T, Cheatham J, Slack M, Rome J (2010) Stenting of aortic coarctation: acute, intermediate, and longterm results of a prospective multi-institutional registry-Congenital Cardiovascular Interventional Study Consortium (CCISC). Catheter Cardiovasc Interv 76(4):553-563

16. Kang S-L, Jivanji S, Mehta C, Tometzki AJ, Derrick $G$, Yates R et al (2017) Outcome after transcathete occlusion of patent ductus arteriosus in infants less than $6 \mathrm{~kg}$ : a national study from United Kingdom and Ireland. Catheter Cardiovasc Interv 90(7):1135-1144
17. Lewis MJ, Kennedy KF, Ginns J, Crystal MA, Torres A, Vincent J, Rosenbaum MS (2016) Procedural success and adverse events in pulmonary artery stenting: insights from the NCDR. J Am Coll Cardiol 67(11):1327-1335

18. LockJE, Bass JL, AmplatzK, Fuhrman BP, CastanedaZuniga W (1983) Balloon dilation angioplasty of aortic coarctations in infants and children. Circulation 68(1):109-116

19. Lock JE, Block PC, McKay RG, Baim DS, Keane JF (1988) Transcatheter closure of VSDs. Circulation 78(2):36-368

20. Malekzadeh-milani $S$, Ladouceur $M$, Patel $M$, Boughenou F-M, Iserin L, Bonnet D, Boudjemline $Y$ (2015) Incidence and predictors of Melody ${ }^{\circledR}$ valve endocarditis: a prospective study. Arch Cardiovasc Dis 108(2):97-106

21. Moore JW, Vincent RN, Beekman RH 3rd, Benson L, Bergersen L, Holzer R, Jayaram N, Jenkins K, Li Y, Ringel R, Rome J, Martin GR (2014) Procedural results and safety of common interventional procedures in congenital heart disease: initial report from the National Cardiovascular Data Registry. J Am Coll Cardiol 64(23):2439-2451

22. O'Byrne ML, Gillespie MJ, Kennedy KF et al (2017) The influence of deficient retroaortic rim on technical success and early adverse events following device closure of secundum atrial septal defects: an analysis of the IMPACTRegistry1. Catheter Cardiovasc Interv 89:102-111

23. O'Byrne ML, Kennedy KF, Rome JJ, Glatz AC (2016) Variability of practice patternsin \& device closure of atrial septal defects and patent ductus arteriosus: an analysis of data from the IMPACT1 registry. Circulation 134(Suppl 1):A15978-A115978

24. Odemis E, Saygi M, Guzeltas A, Tanidir IC, Ergul Y, Ozyilmaz I, Bakir I (2014) Transcatheter closure of perimembranous VSDs usingNit Occlud VSDcoil: early and mid-term results. Pediatr Cardio 35(5):817-823

25. Ogando AR, Asensio IP, Blanca ARS, Tejerizo FB, Luna MS, Jaurena JMG et al (2017) Surgical ligation versus percutaneous closure of patent ductus arteriosus in very low-weight preterm infants: Which are the real benefits of the percutaneous approach? Pediatr Cardiol 39(2):398-410

26. Oses P, Hugues N, Dahdah N, Vobecky SJ, Miro J, Pellerin M, Poirier NC (2010) Treatment of isolated ventricular septal defects in children: Amplatzer versus surgical closure. Ann Thorac Surg 90(5):1593-1598

27. Parent JJ, Ross MM, Bendaly EA, Breinholt JP (2017) Results of pulmonary balloon valvuloplasty persist and improve at late follow-up in isolated pulmonary valve stenosis. Cardiol Young 27(8):1566-1570

28. Portsmann W, Wierny L, Warnke H(1967) Closure of persistent ductus arteriosus without thoracotomy Ger Med Monthly 12(6):259-261

29. Schranz D, Zartner P, Michel-Behnke I, Akinturk $H$ (2006) Bioabsorbable metal stents for percutaneous treatment of critical recoarctation of the aorta in a newborn. Catheter Cardiovasc Interv 67:671-673

30. Scully BB, Morales DL, Zafar F, McKenzie ED, Fraser CD Jr, Heinle JS (2010) Current expectations for surgical repair of isolated ventricular septal defects. Ann Thorac Surg 89:544-549

31. Soulatges C, Momeni M, Zarrouk N, Moniotte S, Carbonez K, Barrea Cet al (2018) Long-term results of balloon valvuloplasty as primary treatment for congenital aortic valve stenosis: a 20-year review. Pediatr Cardiol 36(6):1145-1152 
32. Turner DR, Owada CY, Sang CJetal (2017)Closure of secundum atrial septal defects with the amplatzer septal occluder: a prospective multi-center postapproval study. Circ Cardiovasc Interv 10(8):e4212

33. Yang L, Tai BC, Khin LW, QuekSC (2014) A systematic review on the efficacy and safety of transcatheter device closure of VSDs. JInterv Cardiol 27:260-272

34. Yin S, Zhu D, Lin K, An Q (2014) Perventricular device closure of congenital ventricular septal defects. J Card Surg 29(3):390-400

35. Zartner PA, Neudorf U, Bierbach B, Hart C, Schneider MB (2018) First follow-up of a breakable stent for implantation in infants dedicated for a lifelong stay. Catheter Cardiovasc Interv. https://doi. org/10.1002/ccd.27543

\section{T. Schwenzer, J. Bahm (Hrsg.) \\ Schulterdystokie und Plexusparese}

Klinik, Prävention, Gutachten und Dokumentation

Berlin, Heidelberg: Springer-Verlag 2016, 1. Auflage, 242 S., 43 Schwarz-Weiß-, 36 Farbabbildungen Abb., (ISBN: 978-3-662-48786-0), Hardcover 79,99 EUR

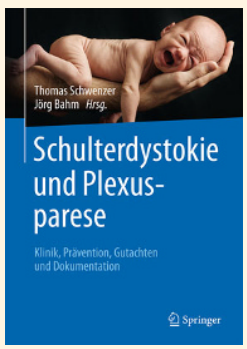

Darf ich über ein Buch, das ich mit Widmung des Erstautors erhalten habe, etwas schreiben? - Ja, vielleicht gerade, weil es einem näher steht als irgendeins.

Thomas Schwenzer beschäftigt sich seit Jahren auch als Autor der Leitlinie zur Schulterdystokie mit diesem schwierigen Thema. Da es jeden Geburtshelfer thematisch betrifft, haben wir uns zu diesem Thema mehrfach ausgetauscht und festgestellt, dass wir nicht in allem dieselbe Meinung vertreten. Während er - auch durch die Zusammenarbeit mit dem Mitherausgeber Jörg Bahn - ein Konzept vertritt, das u.a. auf dem Buch von O'Leary [1] fußt, ist der Rezensent eher ein Anhänger eines kooperativen Ansatzes, wie derjenige der Task Force des ACOG [2]; etwas zurückhaltend in der Kausalzuordnung geburtshilflicher Maßnahmen und Ergebnis beim Neugeborenen.

Das vorliegende Buch ist das Ergebnis jahrelanger Erfahrungen und Auseinandersetzungen in Klinik und Gerichtssaal. Es bietet trotz eines zu knappen Stichwortverzeichnisses den größten deutschsprachigen Fundus an Informationen, die den Anfänger zu überwältigen vermögen.

4 Teile charakterisieren den Inhalt der beiden Ärzte Thomas Schwenzer und Jörg Bahm sowie des Rechtsanwalts Roland Uphoff. Neben "Schulterdystokie" sind dies die Kapitel "Geburtsassoziierte Plexusparese" und „Forensische Fragen bei Plexusparesen und anderen Komplikationen nach Schulterdystokie" sowie abschließend zehn ausgewählte illustrierende Fälle.

Für den Rezensenten stellt dieses Buch eine Zwischenbilanz dar, die eine gute Basis für umfassende Diskussionen ist, die wir nun im deutschsprachigen Raum detailliert führen sollten. Dabei wird es zentral auch darum gehen, bisher apodiktische Ableitungen aus "offensichtlichen", selbstevidenten Befunden mit Evidenz-basierten Ergebnissen aus glaubwürdigen wissenschaftlichen Untersu chungen schrittweise in Einklang zu bringen. Das Thema Schulterdystokie ist keineswegs abgeschlossen, und es wird weiterhin Bemühungen geben müssen, durch Präventivmaßnahmen, durch Schulung, durch Videoanimierte Lehreinheiten und nicht zuletzt durch Hands-on-Training zu einer Verringerung von Problemen beizutragen

Die Schulterdystokie ist Teil des geburtshilflichen Alltags, in dem niemand davor gefeit ist, aktiv in Anspruch genommen zu werden. Acker wies schon vor 30 Jahren klar auf die trotz aller Bemühungen auch in seiner Klinik der Harvard-Universität bestehende Problematik hin: „... however, even the most senior physicians on our staff experienced it." [3] Ja, es ist ein lesenswertes, nützliches Buch, das jedem Geburtshelfer Neues bieten kann und das wenigstens in jede Geburtsklinik gehört, wenn nicht in die Bibliothek jeder geburtshilflich tätigen Person. Allerdings sollte bei all dem Positiven an eine Weisheit erinnert werden, die u.a. Thomas von Aquin zugeschrieben wird: Timeo lectorem unius libri [4].

\section{K. Vetter (Berlin)}

[1] O'Leary, JA (2009) Shoulder dystocia and birth injury. Prevention and treatment. 3rd ed. Totowa, NJ: Humana Press.

[2] Gherman, RB (2014) Neonatal Brachial Plexus Palsy. Report of the American College of Obstetricians and Gynecologists' Task Force on Neonatal Brachial Plexus Palsy. ACOG, Washington. http://sales.acog.org/Neonatal-BrachialPlexus-Palsy-P806.aspx; Summary: ACOG. 2014. "Executive summary: Neonatal brachial plexus palsy. Obstet Gynecol 123 (4):902-4. doi: 10.1097/01.AOG.0000445582.43112.9a.

[3] Acker, DB, Gregory KD, Sachs BP, Friedman EA (1988) Risk factors for Erb-Duchenne palsy. Obstet Gynecol 71 (3 Pt 1):389-392. „... selbst die erfahrensten Kliniker haben Geburten mit betroffenen Kindern geleitet." [4] „Ich fürchte den Leser nur eines einzigen Buches." 